Artículo

\title{
Reporte de Lasiodiplodia theobromae (Pat.) Griffon y Maubl. en árboles cítricos de Tamaulipas
}

\author{
Héctor Flores Hernández ${ }^{1}$ \\ Juan Flores Gracia ${ }^{1}$ \\ Sostenes Edmundo Varela Fuentes ${ }^{2}$ \\ Amado Pérez Rodríguez ${ }^{3}$ \\ Ausencio Azuara Domínguez ${ }^{1}$ \\ Abraham Monteon-Ojeda ${ }^{3 \S}$
}

1Tecnológico Nacional de México-Instituto Tecnológico de Ciudad Victoria-División de Estudios de Posgrado e Investigación. Boulevard Emilio Portes Gil núm. 1301, Ciudad Victoria, Tamaulipas, México. CP. 87010. (floresgracia@yahoo.com.mx; azuarad@ gmail.com). ${ }^{2}$ Universidad Autónoma de TamaulipasFacultad de Ingeniería y Ciencias-Centro Universitario Victoria, Ciudad Victoria, Tamaulipas, México. CP. 87149. (svarela@uat.edu.mx). ${ }^{3}$ Colegio de Postgraduados-Instituto de Fitosanidad. Carretera MéxicoTexcoco km 35.5, Montecillo, Estado de México, México. CP. 56230. (perez.amado@colpos.mx; abraham.monteon@gmail.com).

${ }^{\S}$ Autor para correspondencia: hfhmex@ @otmail.com.

\section{Resumen}

En el presente estudio se determinó la presencia de Lasiodiplodia theobromae en árboles de cítricos con deterioro y muerte descendente en la zona centro de Tamaulipas, México en el 2017 y 2018. En la zona de estudio, se recolectaron muestras vegetales en los árboles de naranja valencia, pomelo, mandarina y limón italiano. En laboratorio, las muestras fueron procesadas y los hongos fueron aislados e identificados con claves taxonómicas y mediante el análisis genético del espaciador interno transcrito y el factor de elongación 1 alfa TEF1. Se aislaron 33 cepas de hongos en las 19 huertas comerciales de cítricos, 26 pertenecieron al género Lasiodiplodia sp., 3 de Botryosphaeria sp., 1 de Colletotrichum sp., 1 de Cyphellophora sp., 1 de Fusarium sp. y 1 de Nigrospora sp. De las cepas del género Lasiodiplodia sp., estas fueron identificadas como $L$. theobromae en árboles de cítricos con gomosis, pudrición, muerte descendente en ramas y momificación de frutos.

Palabras clave: análisis genético, hongos, pudrición

Recibido: febrero de 2021

Aceptado: abril de 2021 


\section{Introducción}

El hongo Lasiodiplodia theobromae (Pat.) Griffon y Maubl. es clasificado dentro de los Ascomicetos, en el orden Botryosphaeriales y familia Botryosphaeriaceae (Schoch et al., 2006; Slippers et al., 2013). Es un hongo saprófito, endófito y se considera patógeno latente. No obstante, es patógeno cuando el hospedero está estresado (Rubini et al., 2005; Mohali et al., 2005).

El hongo provoca la muerte regresiva de las ramas, lesiones en los tallos, genera goma y pudriciones de frutos en post cosecha (Sánchez et al., 1989; Herrera et al., 1993). En campo, en el cultivo de naranja valencia y pomelos ruby red, los daños por $L$. theobromae consisten en: defoliación y presencia de goma en las ramas secundarias, necrosis del floema y xilema.

Por otro lado, también, ocasiona daños al cultivo de mamey [Pouteria sapota (Jacq.) H. E. Moore y Stearn], uva (Vitis vinifera L.), aguacate (Persea americana Mill), kumquat [Fortunella margarita (Lour.) Swingle] y mango (Mangifera indica Lin.) (Úrbez y Gubler, 2011). En estos árboles, L. theobromae puede presentarse solo o en interacción con Colletotrichum sp., Fomitoporia maxonii y Fusarium sp. Dicha interacción provoca clorosis, necrosis, cribados, cancros, tizones, podredumbres húmedas o secas, momificaciones, agallas, costras y marchitez (Kimati et al., 1995).

También, se ha demostrado la interacción de L. theobromae con Fomitiporia maxonii Murrill; Alternaria citri Ellis y Pierce; Colletotrichum gloeosporioides (Penz.) Sacc; Fusarium solanii (Mart.), Appel y Wollen, Fusarium sp, Dothiorella sp, Phytophthora (Oomicetes), Cephaleuros virescens, Kunze) y C. Liberibacter asiaticus (Cabrera et al., 2012, Cabrera et al., 2017). Por lo anterior, en el presente trabajo se determinó la presencia de $L$. theobromae en árboles de cítricos con deterioro y muerte descendente en la zona centro de Tamaulipas, México en 2017 y 2018.

\section{Materiales y métodos}

\section{Área de estudio}

El trabajo de investigación fue desarrollado en el 2017 y 2018 en 19 huertos comerciales de cítricos localizados en el municipio de Güémez, Llera de canales, Padilla y Victoria Tamaulipas, México (Cuadro 1).

Cuadro 1. Localización de los sitios donde se realizó el estudio.

\begin{tabular}{ccccc}
\hline & Localización & & \multicolumn{2}{c}{ Coordenadas } \\
\cline { 1 - 2 } Localidad & Huerta & Latitud & Longitud \\
\hline Güémez & Las Lomas & & 23.920595 & -99.050012 \\
Güémez & Los Cascabeles & & 23.91841 & -99.153615 \\
Güémez & Macabeos III & & 23.923206 & -99.041665 \\
Güémez & Providencia & & 23.927547 & -99.078619 \\
Güémez & Tres Sabinos & & 23.896745 & -99.051205 \\
Llera & Las Angélicas & & 23.249581 & -98.839627 \\
\hline
\end{tabular}




\begin{tabular}{ccccc}
\hline & Localización & & \multicolumn{2}{c}{ Coordenadas } \\
\cline { 1 - 2 } Localidad & Huerta & Latitud & Longitud \\
\hline Llera & La Cecilia & 23.22573 & -98.82675 \\
Padilla & San Juan & & 24.0474 & -99.03125 \\
Padilla & El Tejón & & 24.043433 & -98.891232 \\
Padilla & Macarena & 24.1013 & -99.0193 \\
Padilla & Caluche & & 24.101393 & -99.018491 \\
Victoria & San Francisco & & 23.928958 & -99.232792 \\
Victoria & Real del 14 & & 23.759185 & -99.0723 \\
Victoria & El Huichol & & 23.877121 & -99.238929 \\
Victoria & San José & & 23.903197 & -99.163759 \\
Victoria & El 12 & & 23.827501 & -99.0822 \\
Victoria & Casa Graciela & & 23.896745 & -99.051205 \\
Victoria & El Encino & & 23.78424 & -99.063962 \\
Victoria & El Anhelo & & 23.773975 & -99.07412 \\
\hline
\end{tabular}

\section{Recolecta del material vegetal}

El material vegetal fue colectado en 27 árboles de limón italiano (Citrus limon Burm), 10 de naranja valencia (Citrus sinensis L. Osbeck), 1 de toronja doble rojo (Citrus paradisi Macfad) y 1 de mandarina (Citrus reticulata Blanco). Los árboles presentaban los siguientes síntomas: pudrición del leño, ramas secas, frutos podridos con micelio negro, pudrición y cancros en la corteza de la rama, ramas secas con gomas y hojas con micelio blanco y negro. En cada estructura (leño, rama, fruto y hoja), se recolectaron $200 \mathrm{~g}$ de material. Las muestras fueron vertidas en bolsas de polietileno etiquetadas y transportadas al laboratorio de Biología Molecular y Biotecnología del posgrado en biología del Instituto Tecnológico de Victoria, Tamaulipas.

\section{Aislamiento e identificación taxonómica de los hongos fitopatógenos}

Se cortaron cinco secciones de tejido de $0.5 \mathrm{~cm}$ de cada muestra. Estas fueron desinfectadas con hipoclorito de sodio a $1 \%$ por 3 min, lavadas con agua destilada estéril, secadas y sembradas por separado en papa-dextrosa-agar (Cabrera et al., 2012). Los cultivos fueron incubados a $25^{\circ} \mathrm{C}$ con luz blanca por 3 días. De los aislamientos obtenidos, se realizaron cultivos monospóricos en agua agar (18 g agar disueltos en un litro de agua destilada). Después, los hongos fueron identificados a nivel de género y especie con base en los caracteres taxonómicos publicados por Punithalingam, (1976); Burgess et al. (2006); Barnett y Hunter (2006).

\section{Extracción de ADN y desarrollo de PCR de L. theobromae.}

El ADN se extrajo con la técnica de Ahrens y Seemüller (1992). En el ADN extraído, se amplificó la región genómica ITS (ITS1, $5.8 \mathrm{~S}$ y ITS2) y el gen del factor de elongación alfa (EF-1 $\alpha$ ). La región ITS1 fue amplificada con los iniciadores ITS1 (5'-TCCGTAGGTGAACCTGCGG-3') e ITS4 (3'-TCCTCCGCTTATTGATATGC-5') y la ITS2 con los iniciadores ITS5 (5'GGAAGTAAAAGTCGTAACAAGG-3') e ITS4 (3'-TCCTCCGCTTATTGATATGC-5') (White 
et al., 1990). Mientras que, el EF-1 $\alpha$ fue amplificado con los iniciadores EF1F (5'TGTTGCTGTTAAGGATTTGAAGCG-3') y EF1R (3'-AACAGTTTGACGCATGTCCCT AAC-5') (Rehner y Buckley, 2005).

La mezcla de PCR para amplificar ambas regiones consistió en: agua ultrapura (13.22 $\mu 1)$, solución amortiguadora TBE 1X $(2.5 \mu \mathrm{l}), \mathrm{MgCl}$ a $2.5 \mathrm{mM}(2.08 \mu \mathrm{l})$, dNTPs a $0.2 \mathrm{mM}(2 \mu \mathrm{l})$, iniciadores a $20 \mu \mathrm{mol}\left(2 \mu \mathrm{l}\right.$ de cada uno), DNA polimerasa (Biogenica $\left.{ }^{\circledR}\right)$ a $1 \mathrm{U}(0.2 \mu \mathrm{l})$ y $1 \mathrm{ml}$ de $\mathrm{ADN}(80 \mathrm{ng})$.

Los parámetros para PCR fueron: $94{ }^{\circ} \mathrm{C}$ por $5 \mathrm{~min}, 35$ ciclos de $94{ }^{\circ} \mathrm{C}$ por $5 \mathrm{~min}, 60{ }^{\circ} \mathrm{C}$ por 1 min, $72{ }^{\circ} \mathrm{C}$ por $15 \mathrm{~min}$ y un ciclo final de $72{ }^{\circ} \mathrm{C}$ por $5 \mathrm{~min}$. Las regiones amplificadas fueron purificadas con un kit comercial (Promega) y los fragmentos obtenidos fueron enviados a secuenciar a la Facultad de Ciencias de la Universidad Nacional Autónoma de México (UNAM).

\section{Análisis genético}

Las secuencias obtenidas de la región ITS y del EF-1 $\alpha$ fueron editadas a fin de construir los consensos, alineadas, con el algoritmo Clustal W incluido en el software BioEdit v7.0.9 y comparadas con las secuencias depositadas en el Centro Nacional de Información Biotecnológica (NCBI). Las matrices generadas fueron utilizadas para realizar el análisis filogenético con el método del 'vecino más cercano' basado en el método de Neighbor-Joining (Saitou y Nei, 1987). Seleccionando las secuencias con mayor similitud e igualdad en el tamaño del fragmento a fin de calcular la distancia evolutiva (Tajima y Nei, 1984) en el software MEGA7 (Kumar et al., 2016).

\section{Resultados}

Se determinó la presencia del hongo en los sitios prospectados caracterizando los daños y síntomas en los árboles (Figura 1). Se aislaron 33 cepas de hongos en las 19 huertas comerciales (Cuadro 2). De las cuales, 20 cepas fueron recolectadas en el limón italiano, 11 en naranja valencia, 1 en mandarina y 1 en el cultivo de toronja. En los árboles, 13 cepas fueron recolectadas en el tronco, 13 en las ramas, 3 en frutos, 3 en la raíz y uno en las hojas.

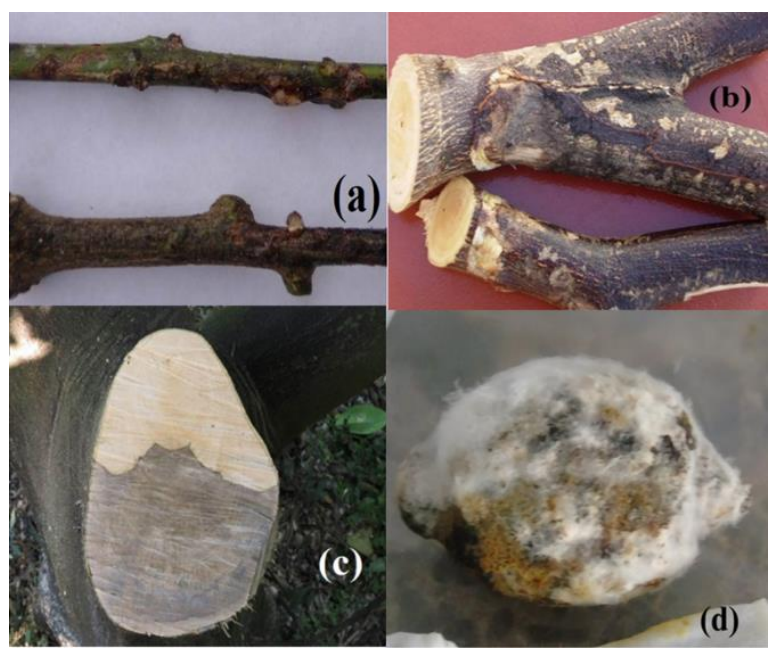

Figura 1. Síntomas de daños en cítricos: gomosis en ramas (a), daño en corteza y leño (b) y (c); pudrición en fruto con crecimiento de micelio (d). 
Cuadro 2. Registro de síntomas en los árboles de cítricos muestreados en la zona citrícola de Tamaulipas, México.

\begin{tabular}{|c|c|c|c|c|}
\hline Municipio & Huerta & Variedad & Estructura & Síntomas \\
\hline \multirow[t]{12}{*}{ Güémez } & Las Lomas & Limón I & Rama & Pudrición y ramas secas \\
\hline & Los Cascabeles & & Rama & Pudrición y ramas secas \\
\hline & & & Tronco & Pudrición del leño y xilema \\
\hline & & Naranja V & Tronco & Pudrición de corteza y leño \\
\hline & & & Rama & Conidios inmaduros \\
\hline & & & Fruto & Frutos podridos \\
\hline & & Limón I & Rama & Micelio negro en el xilema \\
\hline & & & Raíz & Raíz seca y podrida \\
\hline & Macabeos III & & Rama & Pudrición en la rama \\
\hline & & & Raíz & Corteza y xilema dañados \\
\hline & Providencia & & Tronco & Pudrición en leño \\
\hline & Tres Sabinos & & Rama & Rama seca \\
\hline \multirow[t]{2}{*}{ Llera } & Las Angélicas & Naranja V & Tronco & Pudrición del xilema en ramas \\
\hline & La Cecilia & Limón I & Rama & Pudrición del xilema y ramas \\
\hline \multirow[t]{8}{*}{ Padilla } & San Juan & Mandarina & Tronco & Leño seco con afectaciones \\
\hline & & Naranja V & Tronco & Pudrición del leño \\
\hline & & Limón I & Rama & ramas secas con goma \\
\hline & & & Rama & Ramas secas con gomas \\
\hline & El Tejón & Toronja & Tronco & Daños en la xilema \\
\hline & Macarena & Naranja V & Tronco & Pudrición y necrosis del leño \\
\hline & & Limón I & Fruto & Frutos podridos \\
\hline & Caluche & & Tronco & Pudrición del xilema y corteza \\
\hline \multirow[t]{11}{*}{ Victoria } & San Francisco & Naranja & Rama & Ramas secas con goma \\
\hline & Real del 14 & & Fruto & Fruto podrido \\
\hline & & Limón I & Raíz & Raíces secas \\
\hline & El Huichol & & Tronco & Daños en el leño y xilema \\
\hline & & & Tronco & Daños en el xilema y corteza \\
\hline & & & Tronco & Daños en el xilema y leño \\
\hline & San José & Naranja V & Rama & Ramas secas \\
\hline & $\mathrm{El} 12$ & & Rama & Ramas secas \\
\hline & Casa Graciela & & Rama & Rama seca \\
\hline & El Encino & Limón I & Tronco & Xilema dañado \\
\hline & El Anhelo & & Hojas & Hojas con micelio blanco \\
\hline
\end{tabular}


Del total de las cepas recolectadas, 26 pertenecieron al género Lasiodiplodia sp., 3 de Botryosphaeria sp., 1 de Colletotrichum sp., 1 de Cyphellophora sp., 1 de Fusarium sp. y 1 de Nigrospora sp.

De las cepas del género Lasiodiplodia sp., 17 cepas fueron aisladas del cultivo de limón italiano, 7 en naranja valencia, 1 en mandarina y 1 en toronja. En las estructuras de los árboles, 13 cepas fueron recolectadas en el tronco, 10 en las ramas y 3 en los frutos. Mientras que, las tres cepas del género Botryosphaeria sp. fueron recolectadas en las ramas del cultivo de naranja valencia. En contraste, la cepa Colletotrichum sp., Cyphellophora sp. y Fusarium sp., fue recolectada de la raíz de los árboles de limón italiano y la cepa del género Nigrospora sp., fue recolectada en la hoja de esta misma especie de cítrico (Cuadro 3).

Cuadro 3. Distribución de los hongos aislados en las estructuras muestreadas del árbol.

\begin{tabular}{|c|c|c|c|c|}
\hline Municipio & Huerta & Variedad & Estructura & Hongo \\
\hline \multirow[t]{12}{*}{ Güémez } & Las Lomas & Limón italiano & Rama & Lasiodiplodia sp. \\
\hline & Los Cascabeles & & Rama & Lasiodiplodia sp. \\
\hline & & & Tronco & Lasiodiplodia sp. \\
\hline & & Naranja valencia & Tronco & Lasiodiplodia $\mathrm{sp}$ \\
\hline & & & Rama & Botryosphaeria sp. \\
\hline & & & Fruto & Lasiodiplodia sp. \\
\hline & & Limón italiano & Rama & Lasiodiplodia sp. \\
\hline & & & Raíz & Colletotrichum sp. \\
\hline & Macabeos III & & Rama & Lasiodiplodia sp. \\
\hline & & & Raíz & Cyphellophora sp. \\
\hline & Providencia & & Tronco & Lasiodiplodia sp. \\
\hline & Tres Sabinos & & Rama & Lasiodiplodia sp. \\
\hline \multirow[t]{2}{*}{ Llera } & Las Angélicas & Naranja valencia & Tronco & Lasiodiplodia sp. \\
\hline & La Cecilia & Limón italiano & Rama & Lasiodiplodia sp. \\
\hline \multirow[t]{8}{*}{ Padilla } & San Juan & Mandarina & Tronco & Lasiodiplodia sp. \\
\hline & & Naranja valencia & Tronco & Lasiodiplodia sp. \\
\hline & & Limón italiano & Rama & Lasiodiplodia sp. \\
\hline & & & Rama & Lasiodiplodia sp. \\
\hline & El Tejón & Toronja & Tronco & Lasiodiplodia sp. \\
\hline & Macarena & Naranja valencia & Tronco & Lasiodiplodia sp. \\
\hline & & Limón italiano & Fruto & Lasiodiplodia sp. \\
\hline & Caluche & & Tronco & Lasiodiplodia sp. \\
\hline \multirow[t]{4}{*}{ Victoria } & San Francisco & Naranja valencia & Rama & Lasiodiplodia sp. \\
\hline & Real del 14 & & Fruto & Lasiodiplodia sp. \\
\hline & & Limón italiano & Raíz & Fusarium sp. \\
\hline & El Huichol & & Tronco & Lasiodiplodia sp. \\
\hline
\end{tabular}




\begin{tabular}{|c|c|c|c|c|}
\hline Municipio & Huerta & Variedad & Estructura & Hongo \\
\hline & & & Tronco & Lasiodiplodia sp. \\
\hline & & & Tronco & Lasiodiplodia sp. \\
\hline & San José & Naranja valencia & Rama & Botryosphaeria sp. \\
\hline & El 12 & & Rama & Botryosphaeria sp. \\
\hline & Casa Graciela & & Rama & Lasiodiplodia sp. \\
\hline & El Encino & Limón italiano & Tronco & Lasiodiplodia sp. \\
\hline & El Anhelo & & Hojas & Nigrospora sp. \\
\hline
\end{tabular}

Por otro lado, en el caso de las cepas del género Lasiodiplodia sp., las colonias en medio de cultivo a los 10 días desarrollaron un micelio algodonoso color blanco y abundante, posteriormente a los 16 días cambió a un tono gris oscuro (Figura 2) a partir de ahí, estas presentaron paráfisis hialinos, picnidios ostiolados solos o agregados en el tejido estromático, conidios inmaduros hialinos, elipsoides, granulosos y de base truncada. Asimismo, conidios maduros de color café oscuro, elipsoides, con estrías longitudinales y base truncada (Figura 3).

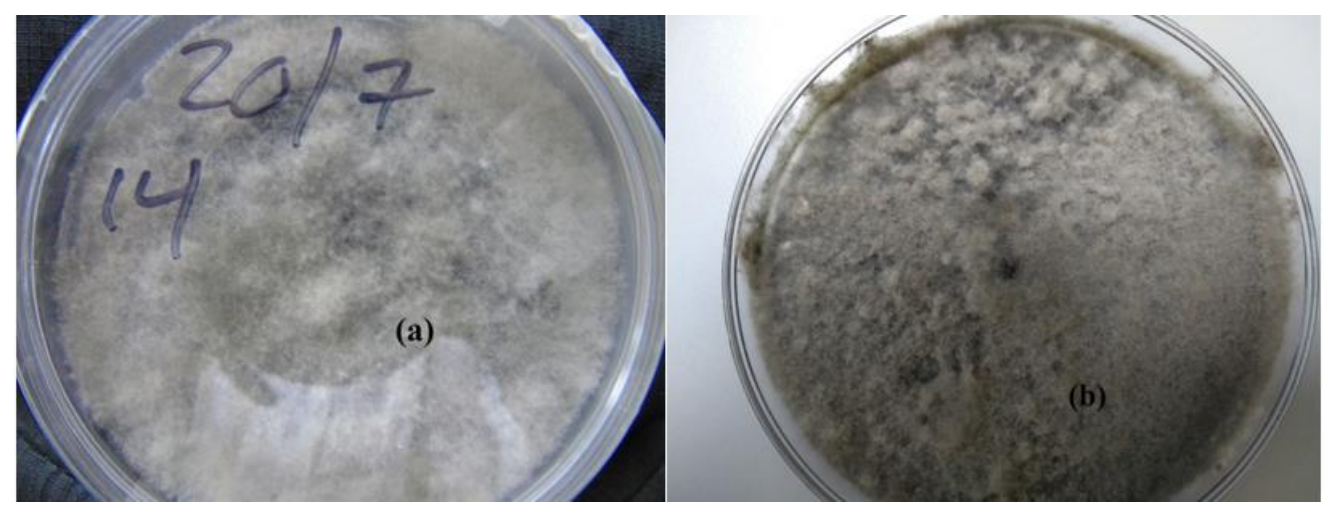

Figura 2. Micelio de Lasiodiplodia sp. (a), a los 10 días el color del micelio es blanco y cubre toda la caja, a los 16 días se tornó a un color gris con centro negro (b).
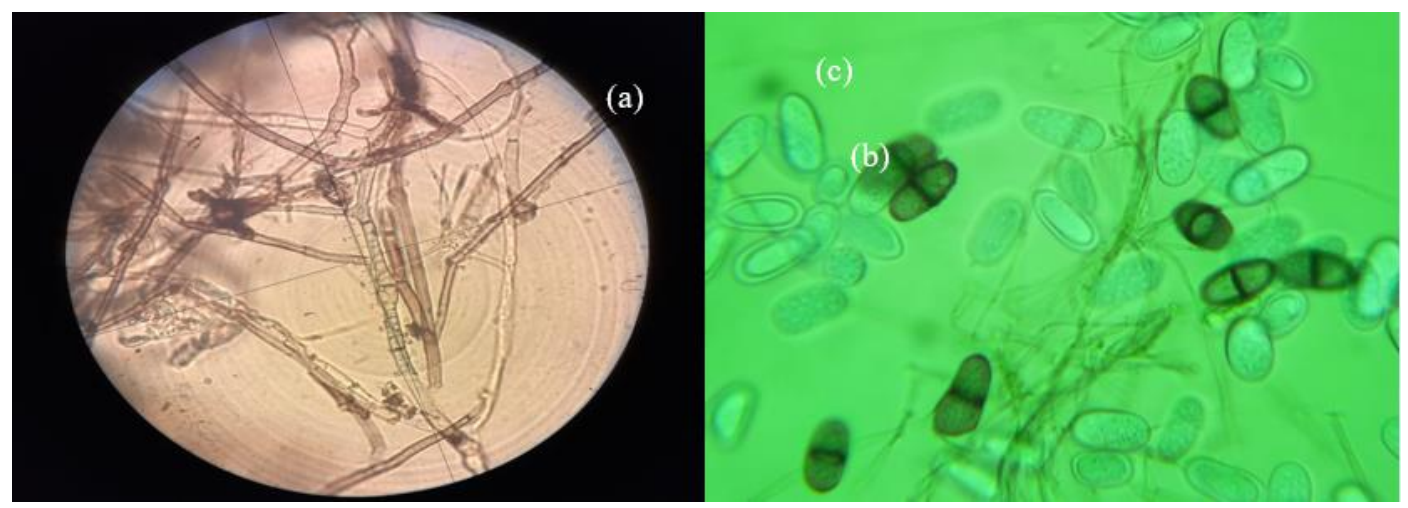

Figura 3. Micelio (a), conidios maduros (b) y jóvenes (c) de Lasiodipodia sp. 


\section{Caracterización genética de las cepas aisladas de $L$. theobromae}

De las cepas aisladas, se obtuvieron 17 secuencias consenso de 542 pares de bases de la región ITS1/5.8S rDNA/ITS2 y 17 secuencias de 314 pares de bases del factor de elongación EF-1 $\alpha$. Las diecisiete secuencias de la región ITS1/5.8S rDNA/ITS2 mostraron homología de 100\% con la especie L. theobromae (HM466958). Estas secuencias no mostraron diferencias genéticas entre si. Por lo anterior, fueron agrupadas en una sola secuencia consenso. Después, esta secuencia fue registrada en el Centro Nacional para la Información Biotecnológica con el acceso MK886711.

Por otro lado, las 17 secuencias del factor de elongación EF-1 $\alpha$ no mostraron diferencias genéticas entre sí. Debido a esto, fueron agrupadas en una sola secuencia de consenso. Después, estas fueron comparadas con secuencias de L. theobromae descargadas del NCBI. La secuencia mostró homología de $100 \%$ con la especie L. theobromae. Finalmente, la secuencia analizada fue depositada en el NCBI con el número de acceso MK531139.

La construcción del árbol filogenético permitió agrupar las 17 secuencias ITS encontradas en ocho grupos o clados de acuerdo con la homología en las secuencias (Figura 4). Mientras que en el árbol filogenético de las secuencias TEF1 alfa, se agruparon en tres clados principales (Figura 5). En ambas construcciones se muestran una estrecha relación genética entre los aislados, Aunque las muestras provenían de diferentes municipios y ejido. La historia evolutiva se infirió utilizando el método de unión de vecinos de Neighbor-Joining. El árbol está dibujado a escala, con la longitud de las ramas en las mismas unidades que las distancias evolutivas para inferir el árbol filogenético.

Las distancias evolutivas se calcularon utilizando el método de máxima probabilidad compuesta. El análisis involucró 17 secuencias de nucleótidos. Las posiciones de codón incluidas fueron $1^{\mathrm{a}}+$ $2^{\mathrm{a}}+3^{\mathrm{a}}$. Se eliminaron todas las posiciones que contenían lagunas y datos faltantes. Hubo un total de 507 posiciones en el conjunto de datos final. Los análisis evolutivos se realizaron en Mega 7.

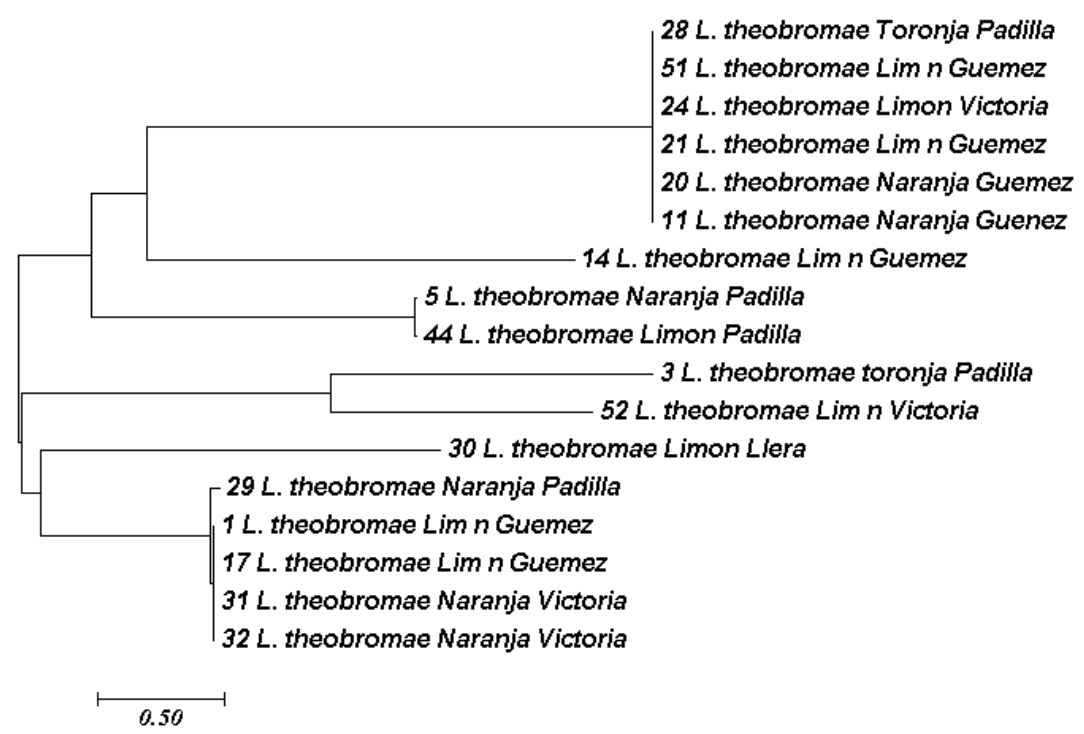

Figura 4. Análisis filogenético generado de las secuencias de la región del ITS ribosomal de las cepas aisladas de $L$. theobromae. El dendograma se obtuvo a partir del análisis por el método del 'vecino más cercano' basado en el método de Neighbor-Joining utilizando el programa Mega 7. 


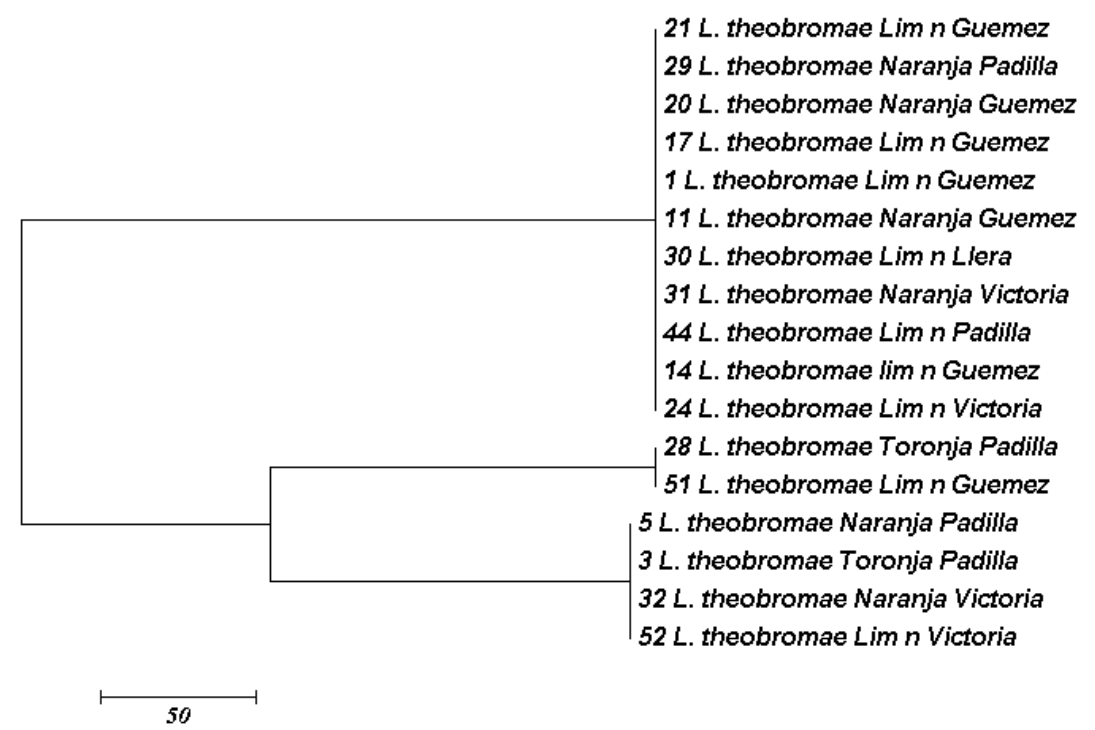

Figura 5. Análisis filogenético generado de las secuencias del factor de elongación EF-1 $\alpha$ del hongo $L$. theobromae. El dendograma se obtuvo a partir del análisis por el método del 'vecino más cercano' basado en el método de Neighbor-Joining utilizando el programa Mega 7.

\section{Discusión}

En diferentes partes del mundo el hongo L. theobromae es reportado en los árboles de cítricos (AlSadi et al., 2014; Adesemoye et al., 2014; Rodríguez et al., 2016). En México, L. theobromae ha sido reportado causando distintas enfermedades en varios cultivos principalmente frutícolas. En el presente trabajo, el hongo fue aislado del cultivo de naranja valencia, limón italiano, mandarina y toronja en el municipio de Güémez, Llera de Canales, Padilla y Victoria Tamaulipas.

En Llera de Canales, Polanco et al. (2019) reporto a este hongo en los árboles de naranja valencia. Mientras que, en el presente trabajo, el hongo fue reportado en el cultivo de limón italiano y naranja valencia en la huerta comercial 'Las Angélicas' y 'La Cecilia'. También estos autores reportaron al hongo en el cultivo de naranja valencia en General Terán y Montemorelos, Nuevo León.

En los sitios donde se ha registrado el hongo, se ha asociado con la muerte regresiva y se ha aislado constantemente de las ramas, corteza, tejido vascular y frutos de las plantas afectadas (Mullen et al., 1991; Moghal et al., 1993; Mohali et al., 2005). Por ejemplo, en Venezuela, L. theobromae se aisló de árboles cítricos con síntomas de muerte regresiva y gomosis (Ferrari et al., 1996). En China, L. theobromae genero gomosis en plantas de Jatropha podagrica (Fu et al., 2007).

Mientras que, en la India este hongo fue el agente causal de la podredumbre en la raíz y de la enfermedad de la podredumbre del collar en J. curcas (Latha et al., 2009). En el presente trabajo, en los árboles de ambas especies de cítricos, el hongo fue recolectado en ramas y troncos con pudrición del xilema, en corteza y ramas secas con presencia de goma. Mientras que, Polanco et al. (2019) lo reportaron en los árboles con síntomas de muerte regresiva y necrosis en el tronco y ramas. Cedeño y Palacios (1992), mencionan que L. theobromae produce gomosis y lesiones en las plantas de cítricos, síntomas parecidos a los observados en el campo. 
Del total de las cepas recolectadas, 26 pertenecieron al género Lasiodiplodia sp., 3 de Botryosphaeria sp., 1 de Colletotrichum sp., 1 de Cyphellophora sp., 1 de Fusarium sp. y 1 de Nigrospora sp. De las cepas del género Lasiodiplodia sp., 17 cepas fueron aisladas del cultivo de limón italiano, 7 en naranja valencia, 1 en mandarina y 1 en toronja. Cabrera et al. (2012) en su estudio describe que las plantaciones de cítricos en estado de estrés y deterioro son importantes fuentes de inóculos de hongos fitopatógenos. Los frutales tropicales son hospedantes de un gran número de estos agentes que causan serios daños en los diferentes órganos de estas plantas, reducen su vida productiva, rendimiento y pueden ocasionar la muerte de estas.

En el caso de la muerte descendente de ramas en cítricos, esta no es una enfermedad exclusiva de este hongo, ya que las especies de $L$. theobromae se presentan de forma conjunta con $N$. mangiferum y $N$. parvum de la familia Botryosphaeriaceae y causan desórdenes de declinación de árboles y pudriciones del pedúnculo del cultivo de mango (Sakalidis et al., 2011).

Además de la interacción con otros hongos fitopatógenos, L. theobromae interacciona con el HLB, Cabrera et al. (2017) sugiere que $C$. liberibacter puede afectar de alguna manera los mecanismos de resistencia o inmunidad de la planta a determinados patógenos como hongos y algas entre otros, y de ahí, desencadenarse la enfermedad como tal. En tal sentido, los resultados infieren que la bacteria pudiera provocar una inmunodeficiencia en las plantas de cítricos. El grado de incidencia de ramas secas parece estar en función del avance de la enfermedad y el grado de debilitamiento de la planta, principalmente en aquellas que manifiestan los síntomas de HLB.

Se ha demostrado la interacción positiva y el deterioro acelerado hasta su muerte, que sufren los árboles de cítricos cuando son afectadas a la vez por HLB y L. theobromae. Se comprobó, mediante ensayos de inoculación de estos hongos en plantas sanas y enfermas con HLB, que las plantas con el hongo y el HLB fueron las más afectadas y exhibían una muerte regresiva más severa (Cabrera et al., 2012). En el presente estudio, L. theobromae fue recolectado en árboles con presencia de la bacteria $C$. liberibacter asiaticus en psílidos; esto acelera el deterioro final del árbol enfermo.

Nariani y Singh (1971), atribuyeron a los hongos C. gloeosporioides; L. theobromae y Fusarium sp. el deterioro acelerado y muerte regresiva de las plantas tras la defoliación causada por HLB. En tal sentido se pudiera considerar que estos patógenos, la mayoría presentes en los cítricos, también ejercen un rol importante en la defoliación inicial. Si se toma en consideración que las plantas afectadas por HLB muestran una abscisión de frutos considerable, con una caída prematura de $60 \%$ al 70\%. Esto permitiría inferir que otros hongos, como los señalados y no solamente la bacteria $C$. liberibacter asiaticus, pudieran ser los máximos responsables de la caída de frutas y de síntomas en plantas con un complejo cuadro patogénico (Gottwald et al., 2012; Cabrera et al., 2017).

\section{Conclusiones}

Los resultados de caracterización sintomatológica, morfológica, análisis de secuencias ITS y factor de elongación $1 \alpha$, determinaron que $L$. theobromae está presente y se asocia a los síntomas de momificación y pudrición de frutos, gomosis, pudrición y muerte descendente en ramas y árboles de diferentes variedades de cítricos, además se detectó a $C$. gloesporoides, C. eucalipti y $F$. keratoplasticum en raíces podridas de árboles con presencia de L. theobromae donde se evidencia una posible asociación con el deterioro de los árboles. Este documento es el primer reporte sobre L. theobromae en la región centro de producción citrícola en Tamaulipas. 


\section{Agradecimientos}

Los autores agradecen al CONACYT por el apoyo otorgado para la realización del presente trabajo de investigación.

\section{Literatura citada}

Adesemoye, A. O.; Mayorquin, J. S.; Wang, D. H.; Twizeyima, M.; Lynch, S. C. and Eskalen, A. 2014. Identification of species of Botryosphaeriaceae causing bot gummosis in citrus in California. Plant Dis. 98(1):55-61.

Ahrens, U. and Seemüller, E. 1992. Detection de DNA of plant pathogenic mycoplasmalike organisms by polymerase chain reaction that amplifies a sequence of the 16S rRNA gene. Phytopathology. 82(8):828-832.

Al-Sadi, A. M.; Al-Ghaithi, A. G.; Al-Fahdi, N. and Al-Yahyai, R. 2014. Characterization and pathogenicity of fungal pathogens associated with root diseases of citrus in Oman. Inter. J. Agric. Bioly. 16(2):371-376.

Barnett, L. H. and Hunter, B. B. 2006. Illustrated genera of imperfect fungi. fourth (Ed). Am. Phytopathol. Soc. St. Paul, Minnesota, USA. 218 p.

Burgess, T. I.; Barber, P. A.; Mohali, S.; Pegg, G.; De Beer, W. and Wingfield, M. J. 2006. Three new Lasiodiplodia spp. from the tropics, recognized based on DNA sequence comparisons and morphology. Mycologia. 98(3):423-435.

Cabrera, R. I.; Betancourt, M.; Ferrer, J.; Hernández, O.; Reyes, N. A.; Piñero, J.; Pérez, V.; Llauger, R. y Herrera, S. 2017. Recuperación de una plantación de naranjo [Citrus sinensis (L.) Osb.] cv. 'Valencia Late' con huanglongbing y otras enfermedades. Rev. Inter. Cítricos. 435(1):71-81.

Cabrera, R. I.; Ferrer; J.; Peña I. y Zamora, V. 2012. Lasiodiplodia theobromae (Pat.) Griffon \& Maubl., sintomatología, afectaciones e impacto en la citricultura cubana actual. Rev. Levante Agríc. 51(412):254-261.

Cedeño, L. y Palacios, P. E. 1992. Identificación de Botryodiplodia theobromae como la causante de lesiones y gomosis en cítricos. Fitopatol. Venezolana. 5(1):10-13.

Ferrari, F. D.; Ochoa, C. F. M. and Subero, M. L. J. 1996. Dieback and gummosis induced by Lasiodiplodia theobromae (Pat.) Griffon and Maubl. on three citrus tree species. Anales de Botan. Agricola. 3(1):46-49.

Fu, G.; Huang, J. G.; Wei, G. Q.; Yuan, J. G.; Ren, W. H. and Cen, Z. L. 2007. First record of Jatropha podagrica gummosis caused by Botryodiplodia theobromae in China. Aust. Plant Dis. 2(1):75-76.

Gottwald, T. R.; Graham, J. H.; Irey, M. S.; McCollum, T. G. and Wood, B. W. 2012. Inconsequential effect of nutritional treatment on huanglongbing control, fruit quality, bacterial titer and disease progress. Cron Protection. 36(6):73-82.

Herrera, L.; Grillo, H.; Pulgarón, A.; Ruiz, B. y Santos, G. 1993. La poda de saneamiento en cítricos. Centro Agrícola. 20(1):33-44.

Kimat, H.; Bergamin F. A. y Amorim, L. 1995. Manual de fitopatologia: princípios e conceitos. Controle químico Editora Agronômica Ceres Ltda. $3^{\text {ra. }}$ Ed. Vol. 1. São Paulo, SP, Brazil. 761-784 p.

Kumar, S.; Stecher, G. and Tamura, K. 2016. MEGA7: molecular evolutionary genetics analysis version 7.0 for bigger datasets. Mol. Biol. Evol. 33(7):1870-1874. 
Latha, P. V.; Prakasam, A.; Kamalakanna, C.; Gopalakrishnan, T.; Raguchander, M.; Paramathma, and Samiyappan, R. 2009. First report of Lasiodiplodia theobromae (Pat.) Griffon and Maubl. causing root and collar rot disease of physic nut (Jatropha curcas L.) in India. Aust. Plant Dis. 4 (2):19-21.

Moghal, S. M.; Shivanathan, P.; Mani, A.; Al-Zidjali, A. D.; Al-Zidjali T. S. and Al-Raeesy, Y. M. 1993. Status of pests and diseases in Oman. Series 1: plant diseases in the Batinah. Muscat: Ministry of agriculture and fisheries. Agricultural Research Center, Rumais First Edition. DGAR, ARC No. 6.

Mohali, S.; Burgess, T. I. and Wingfield, M. J. 2005. Diversity and host association of the tropical tree endophyte Lasiodiplodia theobromae revealed using simple sequence repeat markers. Forest Pathol. 35(6):385-396.

Mullen, J. M.; Gilliam, C. H.; Hagan A. K. and Morgan, J. G. 1991. Canker of dogwood caused by Lasiodiplodia theobromae, a disease influenced by drought stress or cultivar selection. Plant Dis. 75(9):886-889.

Nariani, T. K. and Singh, G. R. 1971. Epidemiological studies on the citrus die- back complex in India. Proc. Indian Natl. Sci. Acad. Ser. 37(5):365-371.

Polanco, L. G.; Alvarado, O. G.; Pérez, O.; González, R. y Olivares, E. 2019. Hongos asociados con la muerte regresiva de los cítricos en Nuevo León y Tamaulipas, México. Rev. Mex. Cienc. Agríc. 10(4):757-764.

Punithalingam, E. 1976. Botryodiplodia theobromae. [Description of pathogenic fungi and bacteria]. Commonwealth Mycological Institute. CAB International CABI Bioscience. No. 52. Bakeham Lane, Egham, Surrey, England. 519 p.

Rehner, S. A. and Buckley, E. 2005. A Beauveria phylogeny inferred from nuclear ITS and EF1$\alpha$ sequences: evidence for cryptic diversification and links to Cordyceps teleomorphs. Mycologia. 97(1):84-89.

Rodríguez, G. E.; Guerrero, P.; Barradas, C.; Crous, P. W. and Alves, A. 2016. Phylogeny and pathogenicity of Lasiodiplodia species associated with dieback of mango in Peru. Fungal Biology. 121(4):1-14.

Rubini, M. R.; Silva-Ribeiro, R. T.; Pomella, A. W.; Maki, C. S.; Araujo, W. L.; dos Santos, D. R. and Azevedo, J. L. 2005. Diversity of endophytic fungal community of cacao (Theobroma cacao L.) and biological control of Crinipellis perniciosa, causal agent of Witches' broom disease. Inter. J. Biol. Sci. 1(1):24-33.

Saitou, N. and Nei, M. 1987. The neighbor-joining method: a new method for reconstructing phylogenetic trees. Mol Biol Evol. 4(4):406-425.

Sakalidis, M. L.; Ray, J. D.; Lanoiselet, V.; Hardy, G. E. StJ. and Burgess, T. I. 2011. Pathogenic Botryosphaeriaceae associated with Mangifera indica in the Kimberley region of western Australia. Eur. J. Plant Pathol. 130(7):379-391.

Sánchez, N.; Zamora, V.; Castellanos, A. y Casín, J. C. 1989. Estudio de hongos encontrados en ramas dañadas por Elaphidion cayamae (Coleoptera: Cerambycidae). Aislamiento y comportamiento en cinco medios de cultivo. Ciencia y Técnica en la Agricultura, Cítricos y otros Frutales. 12(1):131-139.

Schoch, C. L.; Shoemaker, R. A.; Seifert, K. A. Hambleton, S.; Spatafora, J. W. and Crous, P. W. 2006. A multigene phylogeny of the Dothideomycetes using four nuclear loci. Mycologia. 98(6):1041-1052.

Slippers, B.; Boissin, E.; Phillips, A. J. L.; Groenewald, J. Z.; Lombard, L.; Wingfield, M. J.; Postma, A.; Burgess, T. and Crous, P. W. 2013. Phylogenetic lineages in the Botryosphaeriales: a systematic and evolutionary framework. Studies in Mycology. 76(1):31-49. 
Tajima, F. and Nei, M. 1984. Estimation of evolutionary distance between nucleotide sequences. Mol. Biol. Evol. 1(1):269-285.

Úrbez, J. R. and Gubler, W. D. 2011. Susceptibility of grapevine pruning wounds to infection by Lasiodiplodia theobromae and Neofusicoccum parvum. Plant Pathol. 60(2):261-270. 